\title{
Transcutaneous Blepharoptosis Surgery - Advancement of Levator Aponeurosis
}

\author{
Salman Waqar ${ }^{*}, 1$, Catherine McMurray ${ }^{1}$ and Simon N. Madge ${ }^{1,2}$ \\ ${ }^{1}$ West of England Eye Unit, Royal Devon and Exeter Hospital (Wonford), Exeter EX25DS, UK \\ ${ }^{2}$ Hereford County Hospital, Hereford, UK
}

\begin{abstract}
Ptosis surgery has seen many advances in the last few decades, the most important of which have emerged as a result of better understanding of the anatomy and physiology of the eyelid and orbit. Anterior approaches such as a levator aponeurosis advancement, tarsoaponeurectomy and posterior repair involving resection of Muller's muscle have proven to be effective in most cases. The focus of this article is a discussion of the indications, operative techniques, success rates and complications of transcutaneous levator advancement in detail.
\end{abstract}

Keywords: Transcutaneous blepharoptosis surgery, levator aponeurosis, blepharoplasty.

\section{INTRODUCTION}

Ptosis surgery has seen many advances in the last few decades, the most important of which have emerged from a better understanding of the anatomy and physiology of the eyelid and orbit [1]. Patients presenting with symptomatic, involutional levator aponeurotic blepharoptosis most often require surgical repair and anterior approaches such as a levator aponeurosis advancement, tarsoaponeurectomy [2] and posterior repair involving resection of Muller's muscle [3-5] have proven to be effective in most cases. This article will discuss transcutaneous levator advancement in detail.

\section{HISTORY}

The aponeurotic repair approach to ptosis surgery was first described by Everbusch in 1883 [6], however, it did not gain popularity until 1975 when Jones et al. [7] reintroduced it by reporting a series of 57 eyelids of 33 patients. Their technique primarily involved tucking or resecting the flaccid aponeurotic tissue with varied results. A subsequent study by Dortzbach et al. [8] histologically confirmed the presence of a disinserted levator aponeurosis in involutional ptosis and led to various modifications in the techniques originally described by Jones et al. As a consequence of this study, Beard's original classification of acquired blepharoptosis was also modified to include an aponeurogenic category [8].

\section{INDICATIONS}

Blepharoptosis can be classified according to various criteria such as age of onset (congenital or acquired), aetiology, severity and levator function. Acquired blepharoptosis may be further subdivided into myogenic, neurogenic, aponeurotic, mechanical or traumatic causes [8]. Based on severity, it may be minimal or mild (1-2 $\mathrm{mm})$, moderate $(3-4 \mathrm{~mm})$ or severe $(>4 \mathrm{~mm})$ [9]. When considering levator function it can be poor $(0-4 \mathrm{~mm})$, moderate $(5-10 \mathrm{~mm})$ or good $(>10 \mathrm{~mm})[10]$.

\footnotetext{
*Address correspondence to this author at the West of England Eye Unit, Royal Devon and Exeter Hospital (Wonford), Exeter EX25DS, UK; Tel: 07875427149; E-mail: salmanwqr@gmail.com
}

The surgical correction of blepharoptosis must thus be individualised on the basis of degree of ptosis, the levator function and the need for concomitant blepharoplasty or brow surgery. The experience of surgeons and their comfort level with the technique used often determines the procedure of choice [11]. However a fundamental understanding of the underlying anatomical cause of the blepharoptosis can greatly aid in selecting the appropriate surgical procedure.

Aponeurotic ptosis results from an attenuation or disinsertion of the levator aponeurosis from its attachment to the anterior border of the tarsus [12]. Characteristics of aponeurotic ptosis include normal levator function, an elevated eyelid crease and a deep superior sulcus. This is seen most commonly in elderly patients in the form of senile involutional blepharoptosis and such patients are excellent candidates for levator advancement.

\section{OPERATIVE TECHNIQUES}

\section{Traditional Approach}

In the traditional approach, the levator aponeurosis is approached through an upper-eyelid skin incision located at the level of the desired postoperative eyelid crease. This result in severing of the attachments of the levator aponeurosis to the overlying orbicularis muscle and these should be recreated at the end of the procedure in order to ensure an appropriate post-operative lid crease. After appropriate marking, the upper eyelid is typically infiltrated with a small volume of local anaesthetic, typically 1 to 1.5 $\mathrm{ml}$ of 1-2 \% lidocaine mixed with epinephrine, in a subcutaneous plane. The anaesthetic paralyses the orbicularis oculi muscle and the epinephrine can stimulate the Muller's muscle, rendering the intra-operative eyelid position higher than what is expected post-operatively. To avoid this, some surgeons will set the eyelid height 1 to $1.5 \mathrm{~mm}$ higher than the desired post-operative position. Allowing the epinephrine 7 - 10 minutes to work, increases its haemostatic effect allowing better visualisation of tissue planes [11].

A 20 to $22 \mathrm{~mm}$ long lid crease skin incision is then typically made using a No 15 blade but the use of a $\mathrm{CO} 2$ laser has been described [11]. Through the skin incision, 
dissection is carried out superiorly under the orbicularis oculi muscle across the width of the incision. When the orbital septum is identified, it is widely opened to expose the preaponeurotic fat. This is then carefully dissected free of the underlying levator aponeurosis and the upper tarsus is exposed (Figs. 1, 2). Typically, horizontal mattress sutures are then used to advance and reapproximate the levator aponeurosis to the anterior border of the upper tarsus, the choice of suture varying between surgeons (Fig. 3). The first and central suture is typically placed immediately nasal to the pupil, the aim being to create a natural eyelid contour.

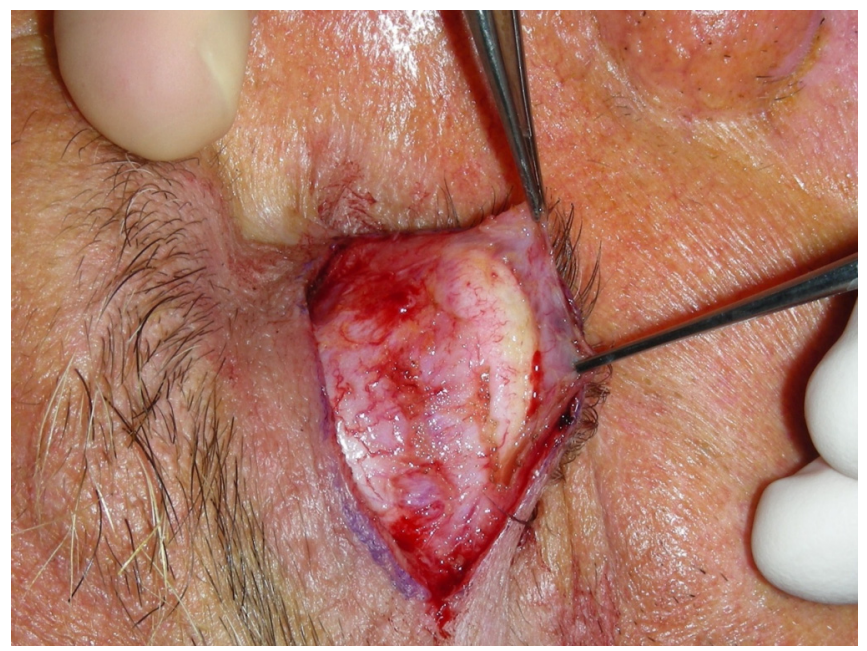

Fig. (1). "Open -sky"approach to combined levator advancement and dermatochalasis repair- Exposure of the tarsal plate.

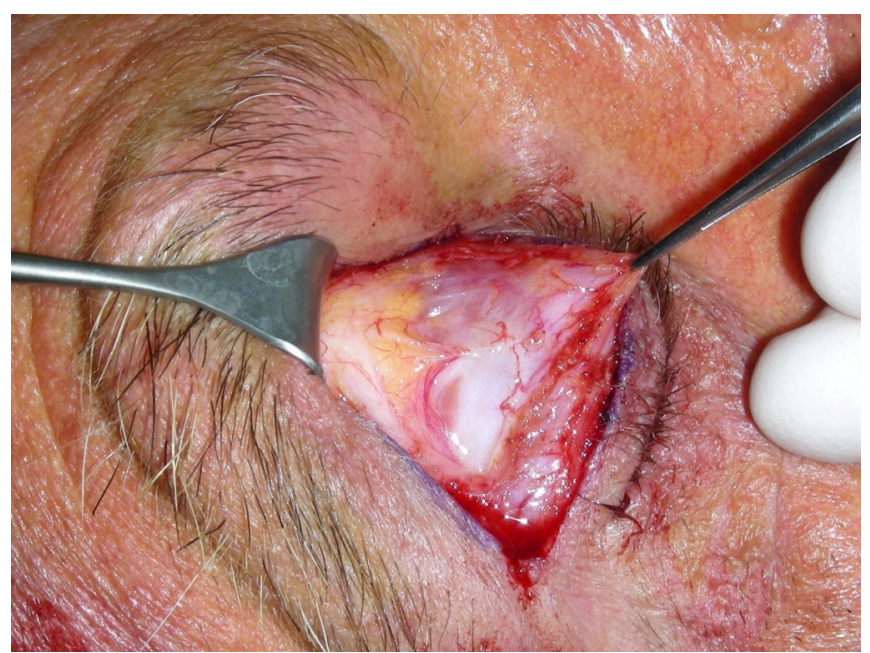

Fig. (2). "Open -sky"approach to combined levator advancement and dermatochalasis repair- Exposed levator aponeurosis under the pre-aponeurotic fat pad.

The overhead theatre lights are now turned off and the patient is asked to open their eyes. After a few blinks, the position (height and contour) of the eyelid are assessed and the central suture is then adjusted until the required eyelid position is achieved (Fig. 4). Additional sutures, typically medially and laterally, are placed by many surgeons to further adjust eyelid contour and to prevent post-operative oedema from altering the eyelid contour [11]. At this stage, a skin and/or muscle blepharoplasty can be performed, typically using scissors to trim the excess tissues according to the preoperative skin markings. A soft eyelid crease can then be created through the placement of absorbable sutures from the pretarsal orbicularis oculi muscle to the distal end of the levator aponeurosis, which may in many instances be preferable to a more 'hard' crease, created using skinaponeurosis-skin sutures. The skin incision is then closed according to preference, typically with a running 6-0 polypropylene suture.

\section{SMALL INCISION MINIMAL DISSECTION APPROACH:}

In line with the current trend in surgery to progressively shift towards minimally invasive procedures, various modifications of levator advancement have been described.

The current trend started with the initial description of the use of a single suture for aponeurotic ptosis correction by Liu [14] in 1993. Meltzer et al. [15] modified this by combining the simplicity of a single suture technique with the flexibility of an adjustable suture and reported excellent results in their retrospective series of 51 patients. The small incision approach was then formally described by Lucarelli and Lemke [16], however, their dissection technique is similar to the traditional approach described above. In their series of patients, 25 of 28 treated eyelids showed satisfactory eyelid position and contour. Baroody et al. [17] reported conducting the procedure in 118 eyelids, of which 105 showed satisfactory marginal reflex distance and eyelid contour. They also noted a decreased incidence of reoperation and postoperative complaints as compared to historical longer-incision cases. Frueh et al. [18] modified the procedure further and reported a retrospective comparative interventional case series in which they noted that the small incision minimal dissection technique is equally effective in correcting eyelid height, superior in producing eyelid contour and being much quicker to perform than the traditional aponeurotic approach.

With the patient in the supine postion on the operating table and looking straight ahead at the ceiling, a vertical line is drawn in line with the centre of the pupil. Another line is drawn in the eyelid crease, centred on the vertical line and 8$10 \mathrm{~mm}$ long. After incision, dissection is performed through the pretarsal orbicularis oculi muscle until the tarsal plate is seen through the semitransparent levator aponeurosis. The aponeurosis is then incised with sharp scissors. Blunt dissection is carried out up under the cut aponeurosis until it is free from the underlying tarsal plate and Muller's muscle to a distance of approximately $12-15 \mathrm{~mm}$. A non-absorbable 6-0 suture is passed through the undersurface of the aponeurosis in this space in line with the vertical lid marking as high as possible and brought out of the upper edge of the incision just posterior to the orbicularis oculi muscle. The two ends of the suture are grasped and held inferiorly with some slack. Overhead lights are dimmed and the patient is asked to open his or her eyes and look up. A firm tug on the suture confirms that the upper extent of the suture has indeed passed through the aponeurosis. The needle is then passed horizontally through the tarsal plate in mid-tarsus centred on the vertical lid mark. A surgeon's knot is placed with an overlying slip knot. The patient is then asked to sit up and open their eyes. The tension in the suture is adjusted until lid height and contour are optimal and the wound is then closed as normal. 


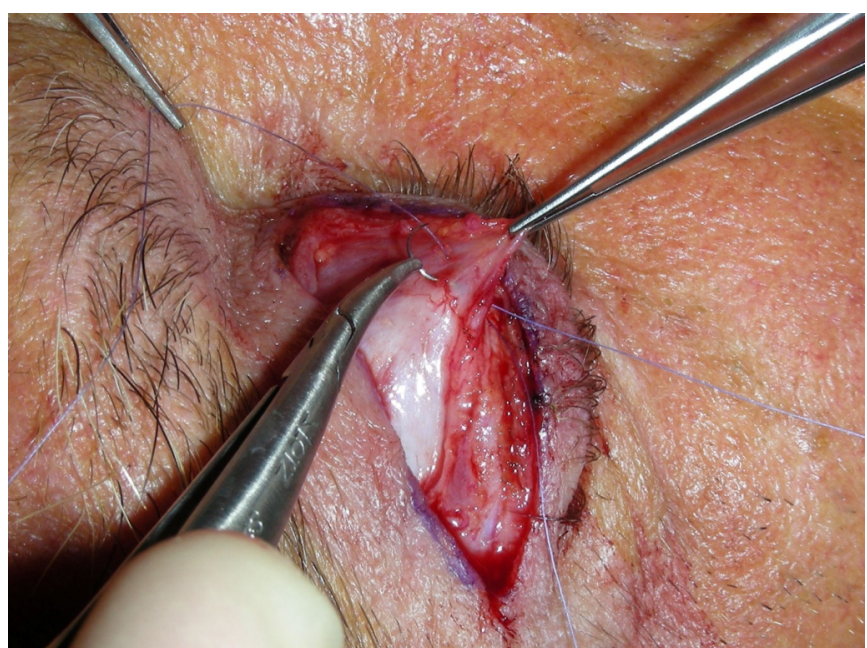

Fig. (3). "Open -sky"approach to combined levator advancement and dermatochalasis repair- Placement of horizontal mattress sutures.

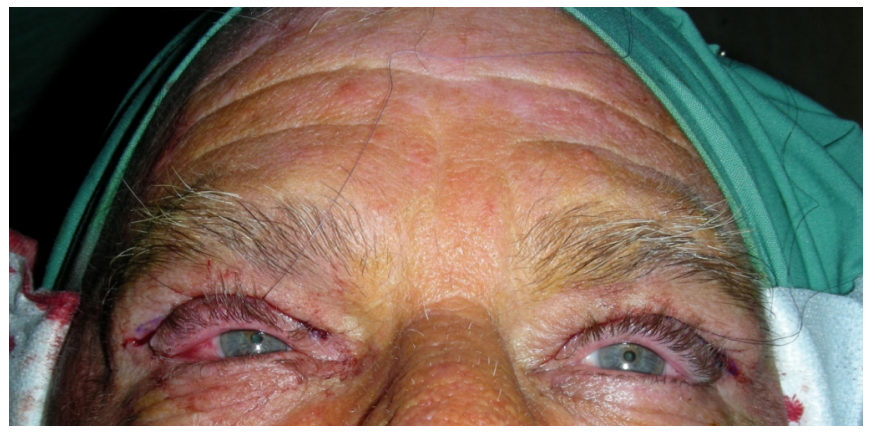

Fig. (4). Intra-operative lid height adjustment with voluntary patient cooperation.

The benefits of the small incision minimal dissection approach are that it is easy to perform and to teach. It lessens anatomical disruption and has a significantly higher rate of good eyelid contour outcome. However, it is only applicable mainly to eyelids that have not had previous lid surgery or trauma, as the anatomy of the lids needs to be in its original state to allow adequate dissection.

A video of the authors demonstrating this technique can be viewed online on the journals website (Video 1)

\section{Three-Step Technique}

Voluntary co-operation of the patient in the operating room to ascertain intra-operative lid height for adjustment can be affected by a variety of factors. Variations in sedation can certainly affect patient co-operation. Local anaesthetic can affect levator function and epinephrine may cause contraction of the Muller's muscle obscuring the true resting lid level. In cases where a general anaesthetic is given, voluntary patient co-operation is of course entirely absent.

Revision rates between 9 to 12 percent have been reported with procedures involving voluntary patient cooperation [18, 19]. More recently McCord et al. [20] have described a three step technique which does not require voluntary patient co-operation. This involves creating a full length lid crease incision and dissecting until the musculoaponeurotic junction is identified. The aponeurosis is incised just above the superior edge of the tarsal plate. After it has been dissected free of the Muller's muscle, a 6-0 double armed suture with small cutting needles is introduced half-thickness within 2-3 mm of the upper edge of the tarsal plate in the exact vertical line of the pupil. The suture is then passed through the musculoaponeurotic junction and tightened until the separation of the upper and lower eyelids is symmetrical between the two eyes. A "spring-back test" is then done to eliminate any difference in suture tension that may cause unequal lid position post-operatively. To do this, the upper eyelid is pulled down by the lashes to the edge of the lower lid and then released. The tension in the sutures adjusted until the velocity of the spring-back is similar in both eyes.

Using this technique, McCord et al. reported a total of 144 procedures on 80 patients with only 14 falling into the criterion for surgical revision (asymmetry between the two eyes greater than $1 \mathrm{~mm}$ or patient dissatisfaction).

\section{Combined Levator Advancement and Blepharoplasty}

Recognition and repair of eyelid ptosis in conjunction with blepharoplasty can make the whole process much more effective and economical [21,22]. Most patients do not present with a complaint of ptosis but have noted or been told that they are looking tired, or that they have excess skin on the upper eyelids without appreciating the ptosis per se. Careful preoperative examination may reveal any combination of brow ptosis, blepharoptosis and dermatochalasis. Better overall cosmetic results may be achieved if a combined levator advancement and blepharoplasty, with or without brow ptosis surgery, is carried out in such patients.

The amount of skin to be excised in a combined procedure needs to be individually determined. In our opinion, the "open-sky" technique of upper blepharoplasty with initial excision of a block of skin and corresponding orbicularis gives the clearest exposure of the levator complex for further blepharoptosis repair (Fig. 5). Once the orbital septum has been opened, the lateral and medial fat pads are easily identified and removed in the usual fashion if desired.

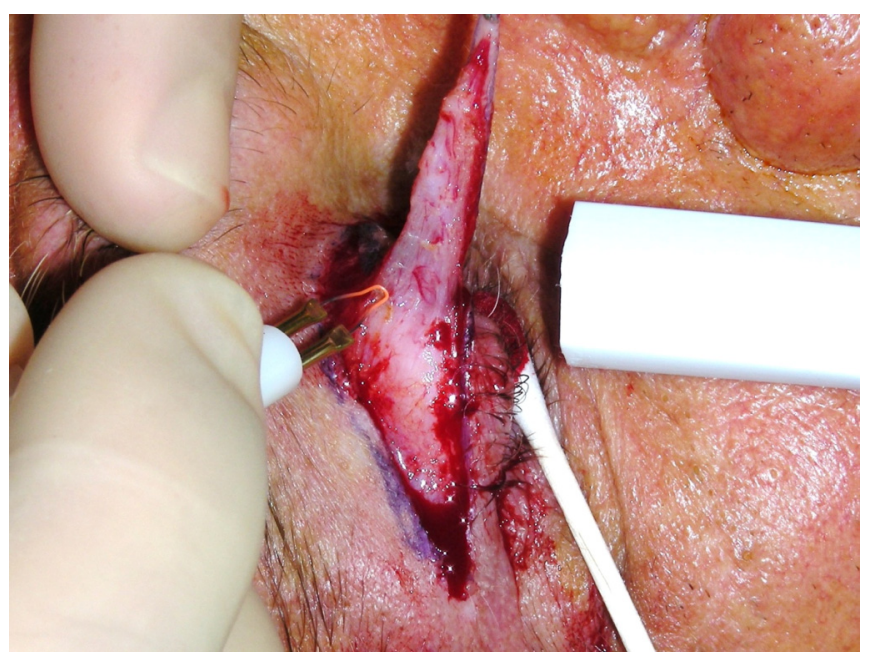

Fig. (5). "Open -sky"approach to combined levator advancement and dermatochalasis repair- Excision of orbicularis muscle. 
Further levator advancement can then be carried out based on the surgeon's preferred technique.

\section{The Use of Local Anaesthesia as Compared to General Anaesthesia}

Most surgeons prefer doing levator advancement under local anaesthesia [23]. This offers the benefit of a "monitored" levator advancement whereby dynamic adjustment of the lid height with voluntary patient cooperation can be done intra-operatively. This advantage is lost while operating under general anaesthesia although McCord et al. three step technique may provide a solution [20].

\section{SUCCESS RATES}

In general, oculoplastic surgery outcomes are inherently less objective and therefore less "measurable" than the majority of other ophthalmic subspecialties and as a result it can be difficult to clearly define criteria for success for any method of blepharoptosis surgery. In recent years it has been widely accepted that a correction of ptosis to within $1 \mathrm{~mm}$ of the desired height is considered successful $[6,19,24]$, which is typically within $1 \mathrm{~mm}$ of the height of the contralateral side. With such a criterion, some patients may be considered as failures when they are actually pleased with their operative result and occasionally patients might not be happy even though the criteria have been met.

The British Oculoplastic Surgery Society (BOPSS) National Ptosis Survey [23] defined an operation as successful only if all of the following criteria were met: upper MRD between 3 and $5 \mathrm{~mm}$, interlid MRD difference 1 $\mathrm{mm}$ or less; interlid crease difference $2 \mathrm{~mm}$ or less; interlid show difference $2 \mathrm{~mm}$ or less and the presence of symmetrical lid contour. In addition, subjective data was collected on patient satisfaction. Although surgeons were free to choose their own desired operative technique, the survey found that levator advancement was used in $87 \%$ of the cases with $82 \%$ of the overall procedures being done under local anaesthetic. The survey reported that $57 \%$ of patients undergoing primary aponeurotic surgery in the UK had a successful outcome with $65 \%$ of patients completely satisfied with the results.

\section{COMPLICATIONS}

As with all surgical procedures, primary prevention of complications in levator advancement should be the goal. The most common complication is unsatisfactory eyelid position or contour. Intra-operative factors producing overcorrection include bright overhead lights causing patient squinting. Excess local anaesthetic can weaken the levator muscle, oedema and haemorrhage can increase the bulk of the eyelid and excess sedation can also affect the eyelid level leading to overcorrection. The effect of epinephrine on Muller's muscle and the weakening effect of local anaesthetic on the orbicularis oculi muscle can cause the intra-operative lid height to appear higher than what is achieved post-operatively. It is important to make the best possible effort to obtain desired position and contour prior to completing the procedure as any discrepancy will seldom resolve spontaneously post-operatively. Post-operative position and contour abnormalities are best managed by early in-office revision as described by Dortzbach and Kronish [25]. Linberg et al. [26] found that the result of ptosis surgery at 3 months after surgery was a good indicator of the long term result and that the lid position at 1 week after surgery predicted the 3 month result accurately.

An orbital compartment syndrome secondary to haemorrhage [11] can be sight-threatening due to increased intraorbital pressure leading to ocular ischemia. Meticulous haemostasis must be maintained during the procedure to avoid this.

All patients should be assessed for tear-film deficiencies prior to the procedure and be warned about the possibility of post-operative irritation if they have a positive Schirmer's test. Relative undercorrection can be considered in such patients.

Postoperative lagophthalmos might result if the orbital septum is incorporated into the wound closure. This should be treated initially with aggressive ocular lubrication and might require lowering of the eyelid, especially if corneal decompensation develops.

Many ptosis surgeons have noticed persistent medial ptosis following an external approach to repair. Kakizaki et al. [27] found in an anatomic study of six Asian cadavers that the medial attachments of the levator are thinner and structurally less dynamic than more lateral attachments, which corresponds with this post-operative observation.

There is also the potential of increased postoperative ptosis in the contralateral eyelid, explicable by Hering's law of equal innervation. Most surgeons believe they can check for the presence of post-operative Hering's law ptosis preoperatively by elevating the ptotic eyelid to watch for a concurrent drop in the contralateral eyelid. However, Erb et al. [28], found a poor correlation between the pre-operative examination for this and the post-operative contralateral eyelid height. Some surgeons advocate that the contralateral eyelid should be corrected at the same time using the same technique to avoid an unpredictable outcome [29]. This remains an area of interest for oculoplastic surgeons and will likely be studied well into the future.

Eyelid crease abnormalities can be avoided by assuring symmetrical placement of the upper eyelid incision and symmetrical excision of redundant skin. Madarosis (eyelash loss) can be avoided by not dissecting within $2 \mathrm{~mm}$ of the lid margin. Epithelial inclusion cysts involving the incision can be avoided by meticulous wound closure without catching the surface epithelium inside the wound.

\section{CONCLUSION}

Levator advancement for the repair of aponeurotic ptosis offers versatility and may be used for all degrees of ptosis provided adequate levator function is present. Small incision approaches offer less surgical trauma with acceptable success rates. Concomitant correction of blepharoptosis and dermatochalasis can be performed, most elegantly in our hands using an 'open sky' approach. Eyelid height and contour can be most easily adjusted intra-operatively under local anaesthesia for the most satisfactory outcomes. Early post-operative adjustment allows eyelid revision with limited additional surgical trauma. 


\section{ACKNOWLEDGEMENTS}

The authors would like to thank Professor D. Selva (Foundation Chair of Ophthalmology, Department of Ophthalmology \& Visual Sciences, University of Adelaide) for allowing use of the video and Dr. Garry Davis for use of the intra-operative photos.

\section{SUPPORTIVE/SUPPLEMENTARY MATERIAL}

Video 1 demonstrating Small Incision Minimal Dissection Technique.

\section{CONFLICT OF INTEREST}

None identified.

\section{REFERENCES}

[1] Anderson R, Beard C. The levator aponeurosis: Attachments and their clinical significance. Arch Ophthalmol 1977; 95: 1437- 41.

[2] McCord CD. An external minimal ptosis procedure: external tarsoaponeurectomy. Trabns Am Acad Ophthalmol Otolaryngol 1975; 79: 683-6.

[3] Fasanella RM. Surgery for minimal ptosis: the Fasanella-Servant operation. Trans Ophthalmol Soc UK 1973; 93: 425-38.

[4] Putterman AM, Urist MJ. Mueller muscle-conjunctiva resection. Arch Ophthalmol 1975; 93: 619-23.

[5] Fasanella RM, Servat J. Levator resection for minimal ptosis: Another simplified operation. Arch Ophthalmol 1961; 65: 55-8.

[6] Liu D. Ptosis repair by single suture aponeurotic tuck: Surgical technique and long term results. Ophthalmology 1993; 100: 251-9.

[7] Jones LT, Quickert MH, Wobig JL. The cure of ptosis by aponeurotic repair. Arch Ophthalmol 1975; 93: 629-34.

[8] Dortzbach RK, Sutula FC. Involutional blepharoptosis: A histopathological study. Arch Ophthalmol 1980; 98: 2045-9.

[9] Tarbet KJ, Lemke BN. Anatomy of the eyelids and lacrimal drainage system. In: Albert DM, Jakobiec FA, Azar Dt, et al., Eds. Princiaples and practice of ophthalmology. Philadelphia: WB Saunders 2000; pp. 3318-32.

[10] Dresner SC. Ptosis management: a practical approach. In: Chen WP, Ed. Oculoplastic Surgery: The essentials. New York: Thieme Medical Publishers 2001; pp. 1-10.

[11] Shovlin JP. The aponeurotic approach for the correction of blepharoptosis. Int Ophthalmol Clin 1997; 37: 1- 3.

[12] Freuh BR, Musch DC. Evaluation of levator muscle integrity in ptosis with levator force measurement. Ophthalmology 1996; 103: 244-50.
[13] Liu D. Ptosis repair by single suture aponeurotic tuck. Surgical technique and long term results. Ophthalmology 1993; 100: 251-9.

[14] Meltzer MA, Elahi E, Taupeka P, Feuer WJ. A simplified technique of ptosis repair using a single adjustable suture. Ophthalmology 2001; 108: 1889-92.

[15] Lemke BN, Lucarelli MJ. Small incision external levator repair: technique and early results. Am J Ophthalmol 1999; 127: 637- 44.

[16] Baroody M, Holds JB, Sakamoto DK, Vick VL, hartstein ME. Small incision transcutaneouys levator aponeurotic repair for blepharoptosis. Ann Plast Surg 2004; 52: 558-61.

[17] Frueh BR, Musch DC, Mc Donald HMB. Efficacy and efficiency of a small- incision minimal dissection procedure versus a traditional approach for correcting aponeurotic ptosis. Ophthalmology 2004; 111: 2158-63.

[18] Shore JW, Bergin DJ, Garrett SN. Results of blepharoptosis surgery with early postoperative adjustment. Ophthalmology 1990; 97: 1502-10.

[19] Mc Culley TJ, Kersten RC, Kulwin TR, Fuer WJ. Outcome and influencing factors of the external levator palpebrae superioris aponeurosis advancement for blepharoptosis. Ophthalmic Plasto Reconstr Surg 2003; 19: 388.

[20] Mc Cord CD, Seify H, Codner MA. Transblepharoplasty ptosis repair: three-step technique. Plast Reconstr Surg 2007; 120: 1037 44.

[21] Carraway JH, Tran P. Blepahorplasty with ptosis repair. Aesthet Surg J 2009; 29: 54-61.

[22] Carraway JH. Combining blepharoplasty with upper eyelid ptosis correction. Aesthet Surg J 2003; 23: 59-62.

[23] Scoppettulol E, Chadha V, Bunce C, Olver JM, Wright M. British Oculoplastic Surgery Society (BOPSS) National Ptosis Survery. Br J Ophthalmol 2008; 92: 1134-8.

[24] Anderson RL, Dixon RS. Aponeurotic ptosis surgery. Arch Ophthalmol 1979; 97: 1123 -8.

[25] Dortzbach RK, Kronish JW. Early revision in the office for adults after unsatisfactory blepharoptosis correction. Am J Ophthalmol 1993; 115: 68-75.

[26] Linberg JV, Vasquez RJ, Chao G-M. Aponeurotic ptosis repair under local anaesthesia: prediction of results from operative lid height. Ophthalmology 1988; 95: 1046-52.

[27] Kakizaki H, Zako M, Ide A. Causes of undercorrection of medial palpebral fissures in blepharoptosis surgery. Ophthalmic Plast Reconstr Surg 2004; 20: 198-201.

[28] Erb MH, Kersten RC, Yip CC, et al. Effect of unilateral blepharoptosis repair on contralateral eyelid position. Ophthalmic Plast Reconstr Surg 2004; 20: 418-22.

[29] Carraway JH. The impact of Herring's Law on blepharoplasty and ptosis surgery. Aesthet Surg J 2004; 24: 275-6. 